\title{
TREATMENT OF SEVERE SPINAL DEFORMATIONS IN CHILDREN WITH IDIOPATHIC SCOLIOSIS USING HALO-GRAVITY TRACTION
}

10.36740/WLek202010107

\author{
Valentyn A. Rohozynskyi ${ }^{1,2}$, Anatolii F. Levytskyi', Mykola M. Dolianytskyi ${ }^{1,2}$, Irina M. Benzar ${ }^{1}$ \\ 'BOGOMOLETS NATIONAL MEDICAL UNIVERSITY, KYIV, UKRAINE \\ 2NATIONAL CHILDREN'S SPECIALIZED HOSPITAL"OHMATDYT", KYIV, UKRAINE
}

\begin{abstract}
The aim: To chose the best way treatment and achievement of 3-dimensional spinal correction in order to maximize its parameters to the physiological norms is a choice of the optimal surgical severe scoliotic spinal deformity correction technology.

Materials and methods: Performed surgical treatments to 60 children of two patient's groups, that were under surgical treatment for severe scoliotic spinal deformations with preliminary used halo-gravity traction and one-step correction; to establish an effective and safe protocol of the treatment for children with severe scoliotic spinal deformations $\left(>100^{\circ}\right)$. The results comparative analysis of 60 patients with severe scoliotic spinal deformations, with a two-step surgery treatments (first step - halo-gravity traction and second step - correcting spinal instrumentation) and patients with one-step correction.

Patients were divided into 2 groups with 30 children in each of them. The first group treated with preoperatively HGT (halo-gravity traction) and after that a spinal instrumentation together with osteotomies (3-4 levels by Ponte; VCR (vertebral column resection) osteotomy 1 level) were performed. The second group - performed one-step spinal instrumentation with osteotomies (3-4 levels by Ponte; VCR osteotomy 1 level).

Results: One-step implanted construction in children with severe scoliotic spinal deformations, compare to HGT treatment that were carried out in stages - is increasing the danger of neurological deficiency by $17 \%$, HGT allows to make more corrections and to adjust spinal cord for the next correction treatment. .

Conclusions: Halo-gravity traction as a first stage of severe scoliotic spinal deformations treatment allows to increase the mobility of the vertebral column and to adjust spinal cord step by step for the next correction treatment.
\end{abstract}

KEY WORDS: vertebral column resection, Ponte osteotomies, severe scoliotic spinal deformations

Wiad Lek. 2020;73(10):2144-2149

\section{INTRODUCTION}

Severe pediatric scoliotic deformations $\left(>100^{\circ}\right)$ make up $0,12-1,7 \%$ of all scoliotic spinal deformations $[1,2]$.

The one-step correction for severe scoliotic spinal deformations in modern surgery practice includes the usage of osteotomies Ponte and Vertebral Column Resection (VCR), which are improving the ability to correct deformations, but also increasing risks of neurological complications, intra-operational blood loss volume. [3,4].

An alternative approach is a preliminary correction of deformation prior to the surgical treatment by applying the Halo-gravity traction. That allows to decrease complications from surgical treatment, to reduce risks of neurological complications and provides a better final spinal correction of the deformation. $[5,6,2]$

Protocols for the surgical treatment of severe spinal deformations are not developed completely and require further study.

\section{THE AIM}

To chose the best way treatment and achievement of 3-dimensional spinal correction in order to maximize its parameters to the physiological norms is a choice of the optimal surgical severe scoliotic spinal deformity correction technology.

\section{MATERIALS AND METHODS}

Orthopedic Traumatology Department of National Children's Specialized Hospital "Ohmatdyt" performed surgical treatments of 60 children with severe scoliotic spinal deformations $\left(>100^{\circ}\right)$ during the period of 2013-2018. Patients were divided into 2 groups with 30 children in each $(25$ male and 35 female).

Patients with idiopathic scoliosis only were included in the research.

All surgical interventions were carried out by the same surgeon using neuro-monitoring.

The following factors were taken into consideration during the research: age, sex, type of spinal deformation, complexity of the surgical interventions, remote surgical treatments results (1-3 years).

The first group treated with preoperatively HGT (halo-gravity traction) and after that a spinal instrumentation together with osteotomies (3-4 levels by Ponte; VCR 
Table 1. Dynamics of the main radiological indicators in the first patient's group ( $n-30)$

\begin{tabular}{cccccc}
\hline № & Indicators & Before & After & 1 year & 3 years \\
\hline 1 & Chest curvature & $108,67 \pm 5,01$ & $47,25 \pm 3,21$ & $46,02 \pm 2,13$ & $44,73 \pm 2,21$ \\
\hline 2 & Lumbar distortion & $69,95 \pm 4,43$ & $28,67 \pm 3,45$ & $27,59 \pm 2,93$ & $28,04 \pm 2,34$ \\
\hline 3 & Chest kyphosis & $64,28 \pm 3,21$ & $49,72 \pm 4,35$ & $47,21 \pm 3,92$ & $48,24 \pm 3,75$ \\
\hline 4 & Lumbar lordosis & $49,15 \pm 2,05$ & $38,24 \pm 2,76$ & $36,11 \pm 3,21$ & $37,31 \pm 3,05$ \\
\hline 5 & Sacral slope & $72,37 \pm 2,24$ & $54,23 \pm 2,66$ & $53,63 \pm 2,23$ & $52,92 \pm 3,11$ \\
\hline 6 & Pelvic Incidence & $41,22 \pm 1,82$ & $34,92 \pm 2,36$ & $31,68 \pm 3,14$ & $32,88 \pm 3,02$ \\
\hline 7 & Pelvic tilt & $9,52 \pm 1,52$ & $9,72 \pm 1,26$ & $9,11 \pm 1,54$ & $9,52 \pm 1,84$ \\
\hline
\end{tabular}

(vertebral column resection) osteotomy 1 level) were performed. The second group - performed one-step spinal instrumentation with osteotomies (3-4 levels by Ponte; VCR osteotomy 1 level).
The research of neurological status was conducted, $\mathrm{X}$-rays (Radiography in standard planes, traction test, tilt test, CT, MRI of the spine). The assessment of the probability of congenital spine and spinal cord diseases was
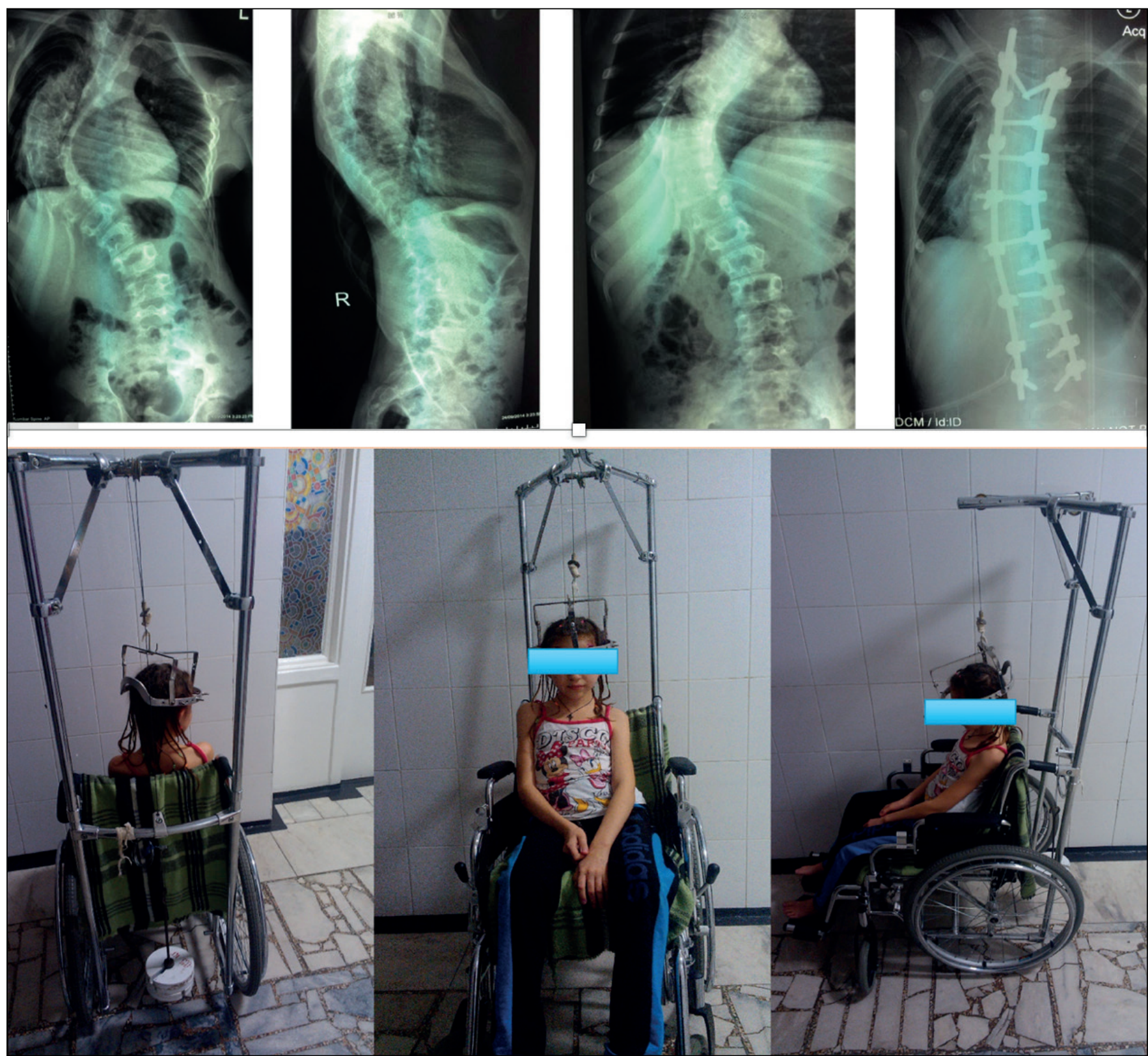

Fig 1. General view and photographic pictures of radiographs of patient K. (12 years old) from the first group with idiopathic lumbosacral scoliosis (major arch 104 degrees before surgery and 43 degrees after posterior instrumentation and osteotomy by Ponte on 4 levels using preliminary halo-gravity traction. 
Table 2. Dynamics of the main radiological indicators in the second patient's group ( $\mathrm{n}-30)$

\begin{tabular}{cccccc}
\hline No & Indicators & Before & After & 1 year & 3 years \\
\hline 1 & Chest curvature & $112,24 \pm 4,23$ & $62,15 \pm 4,41$ & $61,34 \pm 3,01$ & $62,01 \pm 3,54$ \\
\hline 2 & Lumbar distortion & $72,11 \pm 3,63$ & $31,15 \pm 3,84$ & $30,72 \pm 3,26$ & $31,46 \pm 3,15$ \\
\hline 3 & Chest kyphosis & $66,12 \pm 3,77$ & $49,12 \pm 3,93$ & $48,32 \pm 4,02$ & $48,98 \pm 3,88$ \\
\hline 4 & Lumbar lordosis & $47,32 \pm 3,31$ & $39,55 \pm 2,29$ & $40,17 \pm 3,11$ & $39,84 \pm 2,84$ \\
\hline 5 & Sacral slope & $68,21 \pm 3,11$ & $52,42 \pm 3,05$ & $53,15 \pm 3,41$ & $54,21 \pm 4,08$ \\
\hline 6 & Pelvic Incidence & $45,15 \pm 2,07$ & $36,25 \pm 3,18$ & $32,43 \pm 2,96$ & $31,92 \pm 2,97$ \\
\hline 7 & Pelvic tilt & $8,24 \pm 1,52$ & $9,12 \pm 1,54$ & $9,34 \pm 1,71$ & $9,46 \pm 1,71$ \\
\hline
\end{tabular}
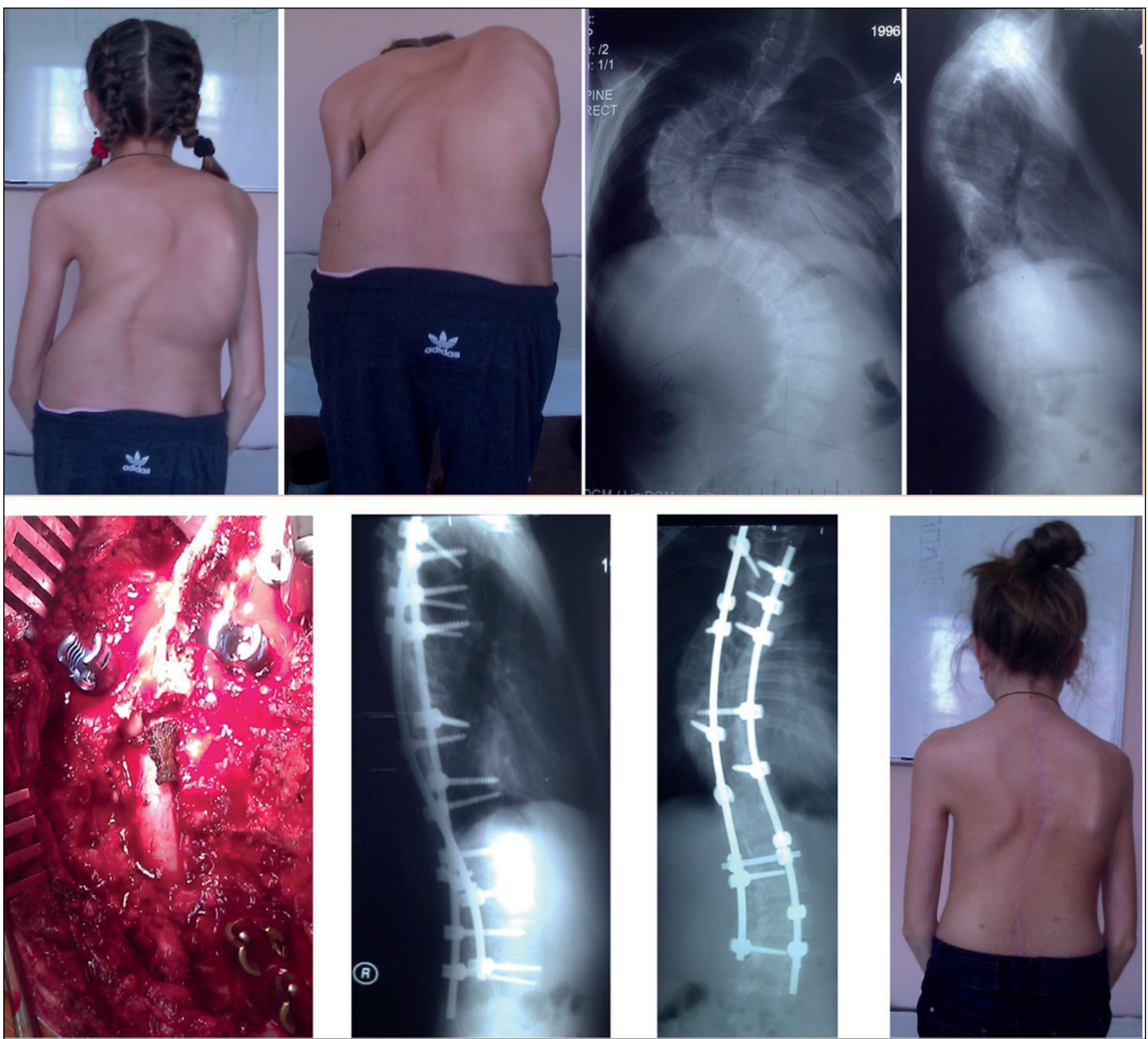

Fig 2. General view and photographic pictures of radiographs of patient 0 . (14 years old) from second group with idiopathic thoracic lumbar scoliosis (major arch was 112 degrees before surgery and 39 degrees after posterior instrumentation and osteotomy by Ponte on 4 levels plus VCR osteotomy on 1 level, that had been conducted a one-step spinal deformations correction.

based on the CT and MRI data. Frontal and sagittal balance assessed by X-ray data, the angle of scoliotic deformation was determined by Cobb.
On the radiographs of the spine in the lateral plane with the capture of the hip joints and a wire temple centered on the spinous sprout C VII in the position of the patient 
standing, determined the magnitude of thoracic kyphosis, lumbar lordosis, obliquity and inclination of the pelvis, inclination of the sacrum. Pathological rotation and torsion of the vertebrae were measured at the apex of the thorasic and lumbar curvatures by using CT. Functional condition of the cardio-respiratory system assessed by using the Spirograph, electroneuromyography of limb muscles (electrophysiological studying methods), electrocardiography, ECHO cardiography.

The amount of trunk decompensation in comparison to sacrum was determined by measuring the distance that moved the pull cord weight.

Halo-gravity traction procedure was performed under full anesthesia in operating room. To minimize the risk of possible instability usually being installed from 6 to 8 screws through halo nimbus. Screws are implanted through a single cortical plate, depending on the size of the patient and the total bone density of the skull. Traction usually started the next day with a minimum weight of 2 to 4 kilograms. Weight was gradually increased from 1 to $2 \mathrm{~kg}$ per day.

The goal was to achieve maximum traction from $33 \%$ to $50 \%$ of body weight, depending on how well it was being tolerated. Traction was applied for a minimum of 12 hours per day. The thrust was performed at wheelchair or inside a standing device. Neurological surveys were conducted every day. Duration of extension was usually from 2 up to 12 weeks, depending on the mobility of the spine. Patients were doing a breathing exercises every day.

Statistical data processing was performed in SPSS 17.0 program.

The study does not contradict the of Helsinki declaration, and received a positive feedback from the Bogomolets National Medical University ethics Commission

\section{RESULTS}

Patients in the first group had been treated with halo- gravity traction to gradually reduce the scoliotic deformations and to prevent neurological disorders. An average duration of spinal traction treatment was $\pm 1,5(\mathrm{P}>0,01)$ month. While conducting halo- gravity traction $40-50 \%$ of the patient's body weight had been used. After halo-gravity traction stage the spine was stabilized with polysegmental structure. An average length of spinal fusion $\pm 11,8$ vertebrae $(\mathrm{P}>0,01)$.

The angle of main deformation in the first group was $108,67 \pm 5,01(\mathrm{P}>0,01)$ in average, in the second group $112,24 \pm 4,23(\mathrm{P}>0,01)$. All the patients had rigid scoliosis deformation: by traction testing the correction of deformation was up to $15-20 \%$. In the first group associated pathology was identified in 7 patients (23,1\%): 4 patients $(13,2 \%)$-infundibular deformity of the chest; 2 patients $(6,6 \%)$ had a congenital club foot; 1 patient $(3,3 \%)$ had cervical deformity of the chest.

In the second group the associated pathology was noted in 10 patients $(33,3 \%): 2$ patients $(6,6 \%)-$ infundibular deformity of the chest; 1 patient $(3,3 \%)$ had a congenital club foot; 4 patients $(13,2 \%)$ had cervical deformity of the chest; 3 patients $(9,9 \%)$ had congenital hip dislocation.

In $92 \% \pm 0(\mathrm{P}>0,01)$ of patients the treatment was conducted at the stage of full grown spine, that was indicated by median of Risser's Sign, CRITOE test, $\mathrm{X}$-ray studies of Ossification of the ulnar process, Taner scale.

In the first group the average age of patients was 13,7 years old \pm 0 . The median for Risser's Sign was 4,2 . ( $P>0,01) .9,9 \%$ of patients at first group had deformations of 1 type by Lenke; $62,7 \%$ had 2 type, $16,5 \%$ had the 3 type; $3,3 \%$ had the $4^{\text {th }}$ type; $6,6 \%$ had the $5^{\text {th }}$ type.

In the second group the average age was 12,6 years old \pm 0 . The median for Risser's Sign was 4,1. ( $>>0,01) .23,1 \%$ of patients at second group had deformations of 1 type by Lenke; $52,8 \%$ had 2 type; $13,2 \%$ had 3 type; $6,6 \%$ had $4^{\text {th }}$ type; $3,3 \%$ had $5^{\text {th }}$ type.

In the first group an osteotomy by Ponte on 4 levels was performed in 18 patients $(59,4 \%)$, and osteotomy by Ponte on 6 levels performed in 10 patients (33\%), and osteotomy by Ponte on 4 levels plus VCR (Vertebral Column Resection) osteotomy on 1 level - in 2 patients $(6,6 \%)$.

For further description of an X-ray indicators of a scoliotic spinal deformations their medians obtained during statistic data processing will be used (Table 1).

At first patient's group an average spinal deformation correction was $57 \pm 12 \%$ (Figure 1). Blood loss during surgery was $20 \pm 10 \%$ (volume of blood in the circulatory system), Cell Saver autogemotransfusion system had been used in all cases. An average duration of surgery was 347 minutes. 2 Patients $(6,6 \%)$ had Transient neurological deficit, 3 patients $(9,9 \%)$ had inflammation of the soft tissues around the core.

In the second patient's group a one-step spinal stabilization by polysegmented structure was performed. An average length of spinal fusion $\pm 12,6$ vertebrae $(P>0,01)$.

In the second group osteotomy by Ponte on 4 levels and VCR osteotomy on 1 level was performed in 14 patients $(46,2 \%)$, osteotomy by Ponte on 5 levels and VCR osteotomy on 1 level - to 11 patients (36,3\%), and osteotomy by Ponte performed on 6 levels - in 5 patients (16,5\%).

For further description of an X-ray indicators of a scoliotic spinal deformations their medians obtained during statistic data processing will be used (Table 2).

In the second patient's group an average spinal deformation correction was $47 \pm 17 \%$ (Figure 2). Blood loss during surgery was $30 \pm 15 \%$ (volume of blood in the circulatory system), Cell Saver autogemotransfusen system was used in all cases. An average duration of surgery was 432 minutes. 6 Patients $(19,8 \%)$ had Transient neurological deficit.

\section{DISCUSSION}

The one-step correction for severe scoliotic spinal deformations in modern surgery practice includes the usage of osteotomies Ponte and Vertebral Column Resection (VCR), which are improving the ability to correct deformations, 
but also increasing risks of neurological complications, intra-operational blood loss volume. [3,4].

An alternative approach is a preliminary correction of deformation prior to the surgical treatment by applying the Halo-gravity traction. That allows to decrease complications from surgical treatment, to reduce risks of neurological complications and provides a better final spinal correction of the deformation. [5,6,2].

Our patients were divided into 2 groups with 30 children in each of them. The first group treated with preoperatively HGT (halo-gravity traction) and after that a spinal instrumentation together with osteotomies (3-4 levels by Ponte; VCR (vertebral column resection) osteotomy 1 level) were performed. The second group - performed one-step spinal instrumentation with osteotomies (3-4 levels by Ponte; VCR osteotomy 1 level).

Between first patient's group was made a poll for subjective analysis on treatment results by SRS-30. Data poll showed more then 800 points, which means that results of the study are good.

Data poll by SRS-30 patients from the second group showed more then 700 points, which means that results of the study are good.

In the course of our work, the scientific goals were achieved in full scope, as evidenced by the results of the study. The lack of a standard approach in the treatment of complex scoliotic deformities of the spine makes this study very promising.

Possible limitations of the current study are patients with a rigid spinal deformities over 100 degrees.

Despite the fact that our patients who used Halo-Gravity Traction were in hospital longer, nevertheless, this is justified by the significantly better clinical and radiological results.

\section{CONCLUSIONS}

One-step implanted construction in children with severe scoliotic spinal deformations, compare to HGT treatment that were carried out in stages - is increasing the danger of neurological deficiency by $17 \%$, HGT allows to make more corrections and to adjust spinal cord for the next correction treatment.

In the surgical treatment of severe scoliotic deformations, HGT reduces the risks of neurological complications, decreases the time of surgery and volume of blood losses, also allows to obtain a better correction results.

The choice of surgical intervention scope was based on anatomical and functional criteria.

\section{REFERENCES}

1. Johnston C.E., Tran D.P., McClung A. Functional and radiographic outcomes following growth sparing management of early-onset scoliosis. J Bone Joint Surg Am. 2017;99:1036 - 42. D0I: 10.2106/JBJS.16.00796.

2. Li X., Zeng L., Li X., Chen X., Ke C. Preoperative halo-gravity traction for severe thoracic kyphoscoliosis patients from Tibet: radiographic correction, pulmonary function improvement, nursing, and complications. Med Sci Monit. 2017;4021-7. D0I: 10.12659/ msm.905358
3. Glotzbecker M., Johnston C.E., Miller P. Is there a relationship betwen thoracic dimensions and pulmonary function in early-onset scoliosis? Spine 2014;39:1590 - 5. DOI: 10.1097/BRS.0000000000000449.

4. Yang C., Zheng Z., Liu H., Wang J., Kim Y.J., Cho S.Posterior vertebral column resection in spinal deformity: a systematic review. Eur. Spine 2016; J 25(8):2368-2375 D0I: 10.1016/j.wneu.2020.03.044

5. Harms L.R., Andras L.M., Sponseller P.D. et al. Comparison of percen- tile weight gain of growth-friendly constructs in early-onset scoliosis. Spine Deform 2018;6:43. D01: 10.1016/j.jspd.2017.05.005.

6. Zhang Z.X., Hui H., Liu T.J., Zhang Z.P. et al Two-staged correction of severe congenital scoliosis associated with intraspinal abnormalities. Clin. Spine Surg. 2016;29(8):E401-405 D0I: 10.1097/BSD.0000000000000175.

7. Johnston C.E.. Halo-gravity traction. In: Akbarnia B.A., Yazici M., Thompson G.H., editors. The growing spine, 2. Berlin: Springer; 2016; 537-51. D01: 978-3-642-43276-7

8. Mehrpour S., Sorbi R., Rezaei R., Mazda K. Posterior-only surgery with preoperative skeletal traction for management of severe scoliosis. Arch Orthop Trauma Surg. 2017;137(4):457-63. D01:10.1007/s00402-0172642-x

9. Janus G.J., Finidori G., Engelbert R.H., Pouliquen M. et al. Operative treatment of severe scoliosis in osteogenesis imperfecta: results of 20 patients after halo traction and posterior spondylodesis with instrumentation. Eur. Spine J. 2000;9(6):486-91. DOI: 10.1007/ s005860000165

10. Koptan W., EIMiligui Y. Three-staged correction of severe rigid idiopathic scoliosis using limited halo-gravity traction. Eur Spine J. 2012;21(6):1091-8. D0I:10.1007/s00586-011-2111-0

11. Yang C., Wang H., Zheng Z., Zhang Z. et al. Halo-gravity traction in the treatment of severe spinal deformity: a systematic review and metaanalysis. Eur. Spine J. 2017;26(7):1810-6. D0I: 10.1007/s00586-0164848-y

12. ZhangZ.X., Hui H.., LiuT.J., ZhangZ.P. etal. Two-staged correction of severe congenital scoliosis associated with intraspinal abnormalities. Clin Spine Surg 2016; 29(8):E401-405 D0I: 10.1097/BSD.0000000000000175.

13. Koller H., Zenner J., Gajic V., Meier 0 . et al. The impact of halo-gravity traction on curve rigidity and pulmonary function in the treatment of severe and rigid scoliosis and kyphoscoliosis: a clinical study and narrative review of the literature. Eur Spine J. 2012; 21:514-529. D0l: 10.1007/s00586-011-2046-5.

14. Koptan W., El Miligui Y. Three-staged correction of severe rigid idiopathic scoliosis using limited halo-gravity traction. Eur Spine 2012;21:10911098. DOI: $10.1007 / s 00586-011-2111-0$

15. Garabekyan T., Hosseinzadeh P., Iwinski H.J., Muchow R.D. et al. The results of preoperative halo-gravity traction in children with severe spinal deformity. J Pediatr Orthop B. 2014;23:1-5. D0I:10.1097/ BPB.0b013e32836486b6.

16. Park D.K., Braaksma B., Hammerberg K.W., Sturm P. The efficacy of preoperative halo-gravity traction in pediatric spinal deformity the effect of traction duration. J Spinal Disord Technol 2015; 26:146-154 D0I: 10.1097/bsd.0b013e318237828c

17. Hui H., Luo Z.J., Yan M., Ye Z.X. et al. Non-fusion and growing instrumentation in the correction of congenital spinal deformity associated with split spinal cord malformation: an early follow-up outcome. Eur Spine J. 2013; 22:1317-1325. D0l: 10.1007/s00586-013-2757-x.

18. Takeshita K., Lenke L.G., Bridwell K.H., Kim Y.J. et al. Analysis of patients with nonambulatory neuromuscular sco- liosis surgically treated to the pelvis with intraoperative halo- femoral traction. Spine 2006;31:2381-5. D0I: 10.1097/01.brs.0000238964.73390.b6 
The work is a fragment of the planned research work of the Department of Pediatric Surgery of the O.O. Bogomolets National Medical University "Surgical Treatment of Severe Spinal Deformations in Children Using Halo-Gravity Traction."

\section{ORCID and contributionship:}

Valentine O. Rohozynskyi: 0000-0001-9891-0739 ${ }^{A, B, D}$ Anatoliy F. Levitskyi: 0000-0002-4440-2090 E,F Mykola M. Dolianitskyi: 0000-0002-0898-6914 ${ }^{\mathrm{C}}$ Irina M. Benzar: 0000-0001-7637-7769 ${ }^{E}$

\section{Conflict of interest:}

The Authors declare no conflict of interest.

\section{CORRESPONDING AUTHOR}

Valentyn A. Rohozynskyi

Bohomolets National Medical University

13 Taras Shevchenko Boulevard, 01601 Kyiv, Ukraine

tel: +380639959795

e-mail:v.rogozinskiy.v@gmail.com

Received: 17.05 .2020

Accepted: 03.09 .2020

A - Work concept and design, B - Data collection and analysis, C - Responsibility for statistical analysis,

D-Writing the article, $\mathbf{E}$-Critical review, $\mathbf{F}$ - Final approval of the article 